\title{
Sintomas de Ansiedade, Angina Pectoris e Diagnóstico de Pacientes Submetidos ao Cateterismo Cardíaco
}

\author{
Eliandra de Paula Cantoni ${ }^{1}$, Michele Marinho da Silveira ${ }^{2}$
}

\begin{abstract}
Resumo: O objetivo desde estudo foi avaliar a relação entre sintomas de ansiedade, angina pectoris e o diagnóstico de pacientes submetidos ao cateterismo cardíaco. Foi utilizada a Escala de Ansiedade de Beck e um questionário sociodemográfico e de saúde. Participaram do estudo 30 sujeitos com idade entre 18 e 65 anos do setor de hemodinâmica de um hospital do interior do Rio Grande do Sul, Brasil submetidos ao procedimento. Os resultados indicaram que $24(80 \%)$ indivíduos apresentam sintomas de ansiedade, não obtendo resultados de significância entre o diagnóstico de extensão da doença, porém 28 (93,3\%) apresentam sintomas de angina, com a angina tipo fisgada a mais relatada $11(36,7 \%)$. Pelo teste qui-quadrado observou-se que há diferença estatisticamente significativa entre angina e o diagnóstico de cinecoronariográfico $(\mathrm{p}=0,008)$. A maior parte dos pacientes com angina não possuía diagnóstico de doenças cardíacas 17 (56,7\%), não apresentaram lesões obstrutivas, mas relatavam sentir angina e ansiedade.
\end{abstract}

Palavras-chave: Ansiedade; Doenças cardiovasculares; Cateterismo cardíaco; Angina pectoris.

\section{Symptoms of Anxiety, Angina Pectoris and Diagnosis of Patients Undergoing Cardiac Catheterization}

\begin{abstract}
This study aimed to evaluate the relationship among symptoms of anxiety, angina pectoris and diagnosis of patients submitted to cardiac catheterization. The Beck Anxiety Scale and a sociodemographic and health questionnaire were used. The study included 30 subjects aged 18-65 years from the hemodynamics sector of a hospital in the interior of Rio Grande do Sul, Brazil, submitted to the procedure. The results indicated that 24 $(80 \%)$ individuals presented anxiety symptoms, and did not obtain significant results between the diagnosis of disease extension, but $28(93.3 \%)$ had angina symptoms, with the most reported angina type $11(36.7 \%)$. From the chi-square test it was observed that there is a statistically significant difference between angina and the diagnosis of coronary angiography $(\mathrm{p}=0.008)$. Most patients with angina had no diagnosis of heart disease $17(56.7 \%)$, had no obstructive lesions, but reported having angina and anxiety.
\end{abstract}

Key-words: Anxiety; Cardiovascular diseases; Cardiac catheterization; Angina pectoris.

\footnotetext{
${ }^{1}$ Graduada em Psicologia pela IMED. Contato: eliandrapaula@yahoo.com.

2 Pós-Doutora em Educação pela Universidade Federal do Rio Grande do Sul. Doutora em Gerontologia Biomédica pela Pontifícia Universidade Católica do Rio Grande do Sul. Mestre em Envelhecimento Humano pela Universidade de Passo Fundo. Graduada em Fisioterapia pela Universidade de Passo Fundo. Professora na IMED campus Passo Fundo - RS no curso de Medicina e Psicologia. Contato: michele.msilveira@gmail.com
} 


\section{Introdução}

O termo doença cardiovascular (DCV) designa uma ampla gama de distúrbios que afetam o coração e os vasos sanguíneos, geralmente em consequência do acúmulo de placas de gorduras nas paredes dos vasos, podendo resultarem em bloqueio total ou parcial da circulação do sangue. As principais doenças cardiovasculares são: infarto agudo do miocárdio (IAM), acidente vascular cerebral (AVC), aterosclerose, doença vascular periférica entre outras (WHO, 2003).

As doenças cardiovasculares são a causa número um de morte em todo o mundo. Estima-se que 17,5 milhões de pessoas morreram de doenças cardiovasculares em 2012, representando $31 \%$ de todas as mortes globais. Dessas mortes, estima-se que 7,4 milhões foram devido à doença cardíaca coronária e 6,7 milhões a acidente vascular cerebral. Mais de três quartos das mortes por DCV ocorrem em países de baixa e média renda. Das 16 milhões de mortes antes dos 70 anos devido a doenças não transmissíveis, $82 \%$ estão em países de baixa e média renda e 37\% são causadas por doenças cardiovasculares (WHO, 2016).

No ano de 2030, em torno de 23,5 milhões de pessoas morrerão de DCVs (WHO, 2012). Por ano, no Brasil é a causa de 308.000 de mortes, associada a fatores de risco como o tabagismo, níveis elevados de colesterol, diabete melito, hipertensão arterial sistêmica, histórico familiar, obesidade, sedentarismo, obesidade central, síndrome metabólica e ingestão de álcool (ARBEX et al., 2010; NASCIMENTO, 2011; NASCIMENTO; FRANCISCO, 2013).

Além disso, a doença arterial coronariana (DAC) constitui-se pelo acumulo de gordura nas artérias coronárias, que são chamadas de placas de ateroma, ou aterosclerose. Com passar dos anos, a placa pode calcificar ou ser rompida, ocorrendo a restrição do fluxo sanguíneo o coração, com esse fluxo lento ou bloqueado, pode avançar para uma isquemia do miocárdio (IAM). Além disso, também ocasionar arritmias cardíacas ou insuficiência cardíaca (SANTOS et al, 2008; TIMERMAN; BERTOLAMI; FERREIRA, 2012; BONOW et al., 2013; MAGALHÃES et al., 2015).

A angina é um dos principais sintomas da DAC, apresentando-se com um desconforto ou dor precordial, caracterizando-se por pressão ou aperto no peito, ocorrendo devido à falta de oxigênio recebido pelo coração (BONOW et al., 2013; TIMERMAN et al.,2012). Os sintomas da angina são relatados, sensação de queimação, dificuldade de respirar, aperto no tórax, com 
irradiação para o ombro esquerdo, braço ou pescoço, aumentando a intensidade, podendo ser que o inicio da dor tenha origem após esforço físico, estresse psicológico e a síndrome coronariana aguda (SCA) (BRAUNWALD et al., 2002).

A apresentação como angina pectoris não se relaciona, significativamente à mortalidade, mas apresenta um sério desconforto e limitações a seus portadores no mundo todo (RUSSELL et al., 2010). Essa dor torácica é umas das principais causas de procura em emergências, com um índice de $15 \%$ a $25 \%$ dos pacientes que apresentam síndrome coronariana aguda. Ocorrem em $2 \%$ dos pacientes podendo levar a consequências graves, IAM e até a morte (BRAUNWALD et al., 2002).

Em vista disso, a angiografia ou cateterismo cardíaco surgiu como um exame para identificar a presença de obstruções arteriais, relacionadas a doenças ateroscleróticas, mensurando a gravidade das variantes patológicas (SCALON et al., 2002). Sua indicação é proposta a pacientes com suspeita de DAC ou com diagnóstico estabelecido de DAC. É uma técnica hemodinâmica diagnóstico-intervencionista que consiste na introdução de um cateter até a aorta e o ventrículo esquerdo por meio de uma punção, podendo ser na artéria, radial, braquial ou femoral com esta via de acesso escolhida pelo médico habilitado, conforme as condições do paciente. A formação de imagens das coronárias se dá por meio da injeção de contraste pelo cateter, procedimento indicado para fins de diagnóstico, havendo a necessidade de exclusão de doença ou definição da extensão da cardiopatia e sua gravidade (LIMA; PEREIRA; CHIANCA, 2006; ROSSATO et al., 2007).

Muitas das manifestações da ansiedade podem estar relacionadas a doenças cardíacas. Os transtornos de ansiedade podem apresentar sintomas como desconforto respiratório, sudorese, taquicardia, dor no peito, palpitação, dor no estômago, dor de cabeça, tonturas, tremores, formigamento, tensão muscular, insônia, medo ou irritação sem motivo aparente, entre outros (DESTÊRRO, 2013). A ansiedade é um sentimento que está na vivência do ser humano, um estado de emoção que aflora no indivíduo em uma determinada ocasião no meio em que ele está inserido. A ansiedade pode se tornar patológica quando ocorre de forma excessiva e desproporcional as necessidades, levando a angústia e sofrimento (AMBAN, 2012).

Os transtornos de ansiedade podem ser mal compreendidos, pois não são reconhecidos, vindo a demonstrar uma lacuna de conhecimento nessa área. A distinção entre sintomas de ansiedade e indícios clinicamente relevantes relacionados a doenças cardíacas, especialmente em pacientes com comorbidade de DCVs, pode ser realmente um desafio. Muitos fatores de 
transtornos psiquiátricos contribuem negativamente sobre a permanência da doença favorecendo para desempenhar um papel de morbimortalidade cardiovascular, pois o paciente tem dificuldade de aderir ao tratamento, afetando a sua qualidade de vida, de forma negativa o ato de decisões clínicas (BRASIL, 2012).

Contudo, observa-se uma carência de estudos, principalmente, sobre o diagnóstico da doença cardíaca, angina sentida, e os sintomas de ansiedade desses pacientes. As manifestações clínicas são bastante relevantes em doenças cardíacas, e não podem deixar lacunas na hora do reconhecimento dos sintomas. Assim, este estudo tem como objetivo relacionar os sintomas de ansiedade com a angina pectoris e o diagnóstico de pacientes submetidos ao cateterismo cardíaco. Para tanto, serão investidas as variáveis sociodemográficas e de saúde dos pacientes, sintomas de ansiedade, tipo de angina pectoris e o diagnóstico de patologia cardíaca dos pacientes submetidos cateterismo cardíaco.

\section{Método}

Tratou-se um estudo descritivo, quantitativo de cunho transversal, com amostra de conveniência em trinta pacientes que foram submetidos ao exame diagnóstico de cateterismo cardíaco atendidos no setor de Hemodinâmica de um hospital na região norte do estado do Rio Grande do Sul, Brasil. Para o cálculo amostral utilizou-se o programa Minitab versão 17 com alpha de 0,05 , poder 0,8 , assumindo o desvio padrão do BAI encontrado na literatura de 9,3 com uma diferença de cinco para uma amostra de 28 sujeitos. $\mathrm{O}$ artigo utilizado investigou a associação entre depressão, ansiedade e qualidade de vida após infarto do miocárdio (LEMOS et al., 2008).

Como critérios de inclusão, indivíduos com idade entre 18 e 65 anos que realizaram o exame de cateterismo cardíaco. Como critérios de exclusão, indivíduos que já tenham sido submetidos à cirurgia cardíaca, indivíduos que não tinham capacidade de comunicação verbal ou escrita e de compreensão do termo de consentimento livre e esclarecido e dos questionários. Dos 32 indivíduos avaliados dois foram excluídos, permanecendo trinta participantes.

Como instrumentos de avaliação foram utilizados: Questionário sociodemográfico e de saúde: questionário, no qual, incluiu as variáveis como sexo, idade, estado civil, escolaridade, 
renda mensal, atividade física, percepção de saúde, uso de medicação, uso de cigarro e bebida (quantidade e frequência), doenças autorrelatadas e tipos de angina pectoris e o diagnóstico de patologia cardíaca (verificados por meio de laudo médico do resultado do exame de cateterismo cardíaco realizado pelo paciente). O Inventário de Ansiedade de Beck (BAI) que auxilia na mensuração da intensidade de ansiedade, é constituído por 21 itens, que são afirmações descritivas de ansiedade que devem ser avaliadas pelos examinados em relação a si mesmo numa escala de quatro pontos, que indicam níveis de gravidade alterados de cada sintoma. $\mathrm{O}$ resultado é feito pelo somatório das respostas dadas pelos examinados nos 21 itens. O maior escore possível é 63. A classificação recomendada para os sintomas de ansiedade é ansiedade mínima (0-7), ansiedade leve (8-15), ansiedade moderada (16-25) e ansiedade grave (26-63). Foi utilizada a versão traduzida e validada para a população brasileira da escala de Beck (CUNHA, 2001).

Esta pesquisa só teve início após a apreciação e aprovação do Comitê de Ética e Pesquisa (CAAE: 59466116.0.0000.5319) e da autorização do hospital na região norte do estado do Rio Grande do Sul, Brasil para coleta de dados. Inicialmente, foi realizada uma triagem entre os pacientes submetidos ao exame e pelos critérios de inclusão e exclusão foram selecionados para participarem da pesquisa. Em seguida, foram individualmente convidados pela pesquisadora que explicou a pesquisa e cada participante preencheu o termo de consentimento livre e esclarecido. Após, o participante respondeu a informações sociodemográficas e de saúde. Ao final, foi aplicada a escala de ansiedade nos pacientes submetidos ao cateterismo cardíaco. Os participantes responderam aos questionários sentados em uma poltrona.

Os dados foram analisados usando o SPSS 23.0 para Windows. As variáveis numéricas foram descritas como média \pm desvio padrão. As variáveis qualitativas foram descritas como frequência absoluta e relativa. Para associação entre variáveis ordinais e nominais (variáveis de desfecho) foi utilizado o teste não paramétrico qui-quadrado, utilizando-se o nível de significância de $5 \%$.

\section{Resultados}

Participaram do estudo trinta sujeitos com média de idade de 55,43 $\pm 5,91$ anos. Na tabela 1 estão apresentadas as características sociodemográficas dos participantes. 
Tabela 1 - Características sociodemográficas dos participantes

\begin{tabular}{ll}
\hline Variáveis & $\mathrm{n}(\%)$ \\
\hline Sexo & $9(30 \%)$ \\
Feminino & $21(70 \%)$ \\
Masculino & \\
Faixa etária & $1(16,7 \%)$ \\
$40-49$ anos & $19(63,3 \%)$ \\
$50-59$ anos & $6(20,0 \%)$ \\
$60-69$ anos & \\
Estado civil & $2(6,7 \%)$ \\
Solteiro (a) & $22(73,3 \%)$ \\
Casado (a) & $4(13,3 \%)$ \\
Separado (a) / Divorciado (a) & $2(6,7 \%)$ \\
Viúvo (a) & $13(43,3 \%)$ \\
Escolaridade & $15(50 \%)$ \\
4 anos de estudo & $1(3,3 \%)$ \\
8 anos de estudo & $1(3,3 \%)$ \\
11 anos de estudo & $1(3,3 \%)$ \\
$\geq 12$ anos de estudo &
\end{tabular}

Fonte: Pesquisa das autoras.

Na tabela 2 estão apresentadas as características de saúde da população em estudo e doenças autorrelatadas.

Tabela 2 - Características de Saúde dos participantes

\begin{tabular}{ll}
\hline Variáveis & $\mathrm{n}(\%)$ \\
\hline Diabetes Melito & \\
Não & $22(73,3 \%)$ \\
Sim & $8(26,7 \%)$ \\
Hipertensão arterial Sistêmica & \\
Não & $12(40 \%)$ \\
Sim & $18(60 \%)$ \\
Dislipidemia & \\
Não & $15(50 \%)$ \\
Sim & $15(50 \%)$ \\
\hline
\end{tabular}




\begin{tabular}{ll}
\cline { 2 - 2 } Obesidade & $23(76,7 \%)$ \\
Não & $7(23,3 \%)$ \\
Sim & \\
Tabagismo & $21(70 \%)$ \\
Não & $2(6,7 \%)$ \\
Sim & $7(23,3 \%)$ \\
Ex-fumante & \\
Etilismo & $23(76,3 \%)$ \\
Não & $7(23,3 \%)$ \\
Sim & \\
Atividade física & $22(73,3 \%)$ \\
Não & $8(26,7 \%)$ \\
Sim & $3(10,0 \%)$ \\
Autopercepção de saúde & $9(30,0 \%)$ \\
Ótima & $15(50,0 \%)$ \\
Boa & $3(10,0 \%)$ \\
Regular & \\
Ruim & $8(26,7 \%)$ \\
Autorrelato de Estresse & $22(73,3 \%)$ \\
Não & $11(27,7 \%)$ \\
Sim & $19(63,3 \%)$ \\
Autorrelato de Depressão & $6(20,0 \%)$ \\
Não & $24(80,0 \%)$ \\
Sim &
\end{tabular}

Fonte: Pesquisa das autoras

Além disso, observou-se que dos pacientes que apresentavam angina, 22 (73,3\%) relataram sentir-se ansiosos, 18 (60\%) deprimidos e 20 (66,6\%) estressados. Contudo, doze (40\%) participantes relataram sentir dificuldade para realizar atividade de vida diária como tomar banho, trocar de roupa, realizar higiene. Dos pacientes que apresentavam angina, 15 (50\%) não tinham diagnóstico de lesão cardíaca, porém seis (20\%) apresentam lesão uniarterial, quatro $(13,3 \%)$ lesão biarterial e três $(10 \%)$ triarterial. Dos pacientes que apresentavam diagnóstico de lesão cardíaca cinco $(16,6 \%)$ tinham angina do tipo fisgada, cinco $(16,6 \%)$ angina tipo queimação, dois $(6,6 \%)$ em facada e um $(3,3 \%)$ em ardência. Dos que não apresentavam lesão cardíaca $15(50 \%)$ relataram sentir angina e dois $(6,6 \%)$ não sentiam angina.

Na tabela 3 são apresentadas as características da angina pectoris e diagnóstico dos pacientes avaliados. 
Tabela 3 - Características da angina e diagnóstico dos participantes

\begin{tabular}{ll}
\hline Variáveis & \multicolumn{1}{c}{$\mathrm{n}(\%)$} \\
\hline Dispnéia & $21(70 \%)$ \\
Sim & $9(30 \%)$ \\
Não & \\
Angina pectoris & $28(93,3 \%)$ \\
Sim & $2(6,7 \%)$ \\
Não & \\
Tipo de Angina & $4(13,3 \%)$ \\
Facada & $11(36,7 \%)$ \\
Fisgada & $10(33,3 \%)$ \\
Ardência & $1(3,3 \%)$ \\
Queimação & $1(3,3 \%)$ \\
Não respondeu & \\
Pré-cordialgia & $18(60 \%)$ \\
Em repouso & $10(33,3 \%)$ \\
Após esforço & $2(6,7 \%)$ \\
Sem dor & $16(53,3 \%)$ \\
Irradiada para membro & $14(46,6 \%)$ \\
Sim & \\
Não & $6(20,0 \%)$ \\
Diagnóstico cinecoronariográfico & $4(13,3 \%)$ \\
Uniarterial & $3(10,0 \%)$ \\
Biarterial & $17(56,7 \%)$ \\
Triarterial &
\end{tabular}

Fonte: Pesquisa das autoras

A média encontrada da fração de ejeção que é a força do coração foi de $54,14 \pm 13,8 \%$ e a média de sintomas de ansiedade verificados pelo BAI foi de 20,40 $\pm 10,36$ pontos representando um grau de ansiedade moderada, com uma pontuação mínima encontrada de quatro pontos e máxima de 42 pontos. Quando se analisou a angina em relação aos sintomas de ansiedade (BAI), não se observou diferença estatisticamente significativa nesta condição $(\mathrm{p}=0,764)$. Também não se observou diferença estatisticamente significativa entre os sintomas de ansiedade e o diagnóstico de doenças cardiovasculares $(\mathrm{p}=0,535)$, mas se verificou diferença estatisticamente significativa entre angina e o diagnóstico de cateterismo cardíaco $(p=0,008)$.

\section{Discussão}

Há poucos dados empíricos sobre sintomas de ansiedade, diagnóstico cinecoronariográfico e angina pectoris. Quando se trata de cateterismo cardíaco e ansiedade 
existem estudos internacionais, como Van Gestel et al. (2007), Pedersen et al. (2008), Nilsson et al. (2009) e Poliwczak, Funt e Broncel (2013), mas não são encontrados estudos que relacionem a ansiedade, o diagnóstico de doença cardíaca e angina pectoris.

Como resultado, inicialmente, procurou-se caracterizar o perfil dos pacientes que realizaram cateterismo cardíaco, dos 30 indivíduos a maioria era do sexo masculino 21 (70\%) o que não corrobora o estudo de Schmidt et al. (2011) que avaliou 86,7\% mulheres com sintomas de ansiedade e de depressão. Quanto à faixa etária a mais prevalente foi com pacientes entre 50 a 59 anos o que se assemelha com outra pesquisa em que a maioria dos entrevistados encontrava-se na faixa etária entre 51 a 67 . A maioria dos participantes deste estudo apresentava de quatro a oito anos de estudo corroborando com Matozinhos et al, (2017) que relata que a incidência de doenças cardíacas prevalece em pessoas com nível de escolaridade baixa. Outra pesquisa de Machado, Pires e Lobao (2012) realizada no Brasil revela que quanto menor a escolaridade e as situações socioeconômicas, maior é o fator de risco pré-dispondo às doenças cardiovasculares, favorecendo a hábitos como tabagismo, alcoolismo, sedentarismo, elevando a progressão de doenças cardíacas.

Com relação ao estado emocional relatado pelos pacientes a maioria sentia-se com humor deprimido, estressados e com sintomas de ansiedade moderado, diferentemente de outro estudo em pacientes submetidos ao cateterismo cardíaco que observou grau de ansiedade da maioria dos pacientes de mínimo a leve. Como um dado relevante neste estudo observou um alto índice de pacientes se autorrelatando com sintomas depressivos, verificando em outros estudos que evidencias de fatores psicossociais contribuem para desenvolvimento da DAC. Assim, pessoas depressivas têm maior o risco de desenvolver angina e IAM comparadas as pessoas não depressivas, além disso, tanto a depressão quanto a DAC podem levar a desencadear um processo inflamatório (STRIK; STEPTOE, 2002).

Com relação às características de saúde dos participantes, apenas sete $(23,3 \%)$ já foram fumantes e dois $(6,7 \%)$ continuam fumando. Conforme Ferreira (2000) uma vez que o risco do desenvolvimento de doenças coronarianas é maior em tabagistas e na faixa etária entre 45/65 anos o risco triplica. A pesquisa demonstrou que o uso do álcool está presente em apenas 7 $(23,3 \%)$ dos participantes, outro pesquisador revelou que as taxas de álcool têm efeito tóxico sobre o coração podendo levar a miocardiopatia alcoólica.

A hipertensão arterial sistêmica é reconhecida como o principal fator de risco para a morbidade e mortalidade precoces causadas por doenças cardiovasculares (REZA; 
NOGUEIRA, 2008). A maior parte dos pacientes nesta pesquisa eram hipertensos 18 (60\%) e apresentavam dislipidemia $15(50 \%)$ e apenas $8(26,7 \%)$ tinha diabetes corroborando os achados do estudo de Gus, Fischmann e Medina (2015) estudo que relatou que apesar do número de pacientes fumantes e sedentários diminuir, a hipertensão arterial sistêmica, dislipidemia e a obesidade aumentaram na população, sendo a obesidade um fator de risco mais encontrado nas mulheres e a hipertensão nos homens.

Quanto à autopercepção de saúde, 15 (50\%) participantes apresentaram saúde regular e $22(73,3 \%)$ relataram não praticar atividades física, revelando que os mesmos apresentam um hábito de vida sedentário confirmando os achados com outra pesquisa de Gus et al. (2015) que constatou o sedentarismo como um fator de risco apresentando $70 \%$ dos sujeitos sem praticar algum tipo de atividade física. Segundo Fonseca (2000), a atividade física pode diminuir a vulnerabilidade para requerimento de oxigênio para o miocárdio e complicações cardíacas, ou seja, praticar exercícios físicos significa promover saúde. Sardinha e colaboradores (2011) relatam que pessoas com sintomas de ansiedade geralmente não tem um estilo de vida saudável, não tem uma dieta equilibrada contribuindo para o desenvolvimento de DAC. Além disso, é evidenciado que os sintomas de ansiedade e depressão são menos graves em homens do que em mulheres com a doença.

Dos 30 pacientes avaliados por suspeita de doença coronária submetidos ao cateterismo cardíaco, 13 (43,3\%) apresentaram lesão coronariana significativa, podendo ser uniarterial, biarterial e triarterial, consideradas com estenose acima de $75 \%$, em qualquer artéria coronária. Além disso, $17(56,7, \%)$ dos pacientes apresentaram coronárias sem lesão obstrutiva, em alguns casos apresentam irregularidades podendo no futuro estabelecer a doença. Em vista disso, Bonow et al. (2013) revela que a DAC caracteriza-se pelo acúmulo progressivo de placa de gordura nas coronárias que com passar dos anos é chamado de aterosclerose, podendo tornar-se endurecida, ou ser rompida, causando redução do fluxo sanguíneo, classificando como estenose, tendo como resultado um quadro de isquemia ou IAM.

Com relação à fração de ejeção (FE) a média encontrada foi de 54,14 \pm 13,8\% corroborando o estudo de Shmidt et al. (2011) onde as características clínicas e angiográficas encontradas da fração de ejeção foi de $63 \%$. vs 66\% conforme as análises angiográficas. Quanto aos sintomas de ansiedade, outros pesquisadores que apontam que a ansiedade tem relação com o desenvolvimento de eventos cardíacos (ROEST et al., 2010). Neste estudo, verificou que a população avaliada apresenta nível moderado de ansiedade, mas que não houve diferenças 
estatisticamente significativas entre os sintomas de ansiedade (BAI), diagnóstico cinecoronariográfico e angina, evidenciando que a maioria dos pacientes que teve nível de ansiedade moderada, mas não evidenciou doença, mesmo que 93,3\% apresentaram sintomas de angina. Bonow et al. (2013) ressalta que a angina, ou seja dor ou desconforto no peito (pressão ou aperto) é um dos principais sintomas da DAC, indicando que o coração não está suficientemente oxigenado, parâmetro este de grande importância para o diagnóstico.

Também se percebe que os sintomas de DAC e ansiedade muitas vezes são confundidos, e esses pacientes recorrem a serviços de emergência por angina, vômito, sudorese, sensação de morte, que são sintomas compartilhados nas duas patologias (ansiedade e DAC), ressaltando a importância das equipes terem entendimento adequado dos sintomas de ansiedade para melhor atender o paciente (ZWIELESKI; BUB, 2014). Contudo, um estudo recente apontou que pacientes que tiveram duas ou mais hospitalizações por DAC e internaram novamente, manifestaram um grau de ansiedade mais elevado, ou seja, pacientes com várias internações apresentando ansiedade, tem mais risco de reinfarto e morte (CIRIC-ZDRAVKOVIC et al., 2014).

\section{Conclusões}

Por fim, os resultados obtidos neste estudo esclarecem alguns questionamentos iniciais que indicaram que os pacientes submetidos ao cateterismo cardíaco apresentam sintomas de ansiedade moderado, não obtendo resultados de significância entre o diagnóstico de extensão da doença, porém que maioria apresenta sintomas de angina é do tipo facada, demonstrando que há diferença estatisticamente significativa entre angina e o diagnóstico de cateterismo cardíaco.

Contudo, este trabalho teve a intenção de proporcionar uma reflexão em relação aos sintomas de ansiedade, a prática da atenção em saúde em pacientes com sintomas de DAC, buscando, de certo modo, resgatar a visão em relação mente e corpo, compreendendo as enfermidades como conflitos existências e não fragmentando o ser humano. $\mathrm{O}$ fator emocional tem relação direta com a doença do coração também por seu simbolismo, necessita ser levada em conta a singularidade no processo do adoecer, tornando mais adequada e humana as 
necessidades do indivíduo. Pode-se concluir que os pacientes com angina, sem um diagnóstico de doença cardíaca foram os que mais apresentaram ansiedade.

Assim, sugerem-se novos estudos que irão contribuir para um melhor entendimento entre os sintomas de angina, de ansiedade e o diagnóstico de patologia cardíaca. Percebe-se uma carência da atuação do psicólogo nesta área, a falta de atendimento multidisciplinar, que favoreça a qualidade de vida do paciente.

\section{Referências}

AMBAM, Programa Ansiedade. Transtornos de ansiedade. 2012. Disponível em: http://www.amban.org.br/content/textos-educativos

ARBEX, M. A., et al. Impact of outdoor biomassa in pollution on hypertension hospital admissions. Epidemiol Community Health, v. 64, n. 7, 573-579, 2011.

BARLOW, D. H. Psicopatologia: uma abordagem integrada (4a ed.). São Paulo: Cengage Learning, 2010.

BONOW, R. O. et al. Braunwald - Tratado de doenças cardiovasculares (9a ed.). Rio de Janeiro: Elsevier, 2013.

BRASIL. Ministério da Saúde. Secretaria de Atenção à Saúde. Departamento de Atenção Básica. Acolhimento à demanda espontânea: queixas mais comuns na Atenção Básica / Ministério da Saúde, Secretaria de Atenção à Saúde, Departamento de Atenção Básica. - Brasília: Ministério da Saúde, v. 2. n. 28, p. 01-290. 2012.

BRAUNWALD, E. et al. ACC/AHA guideline update for the management of patients with unstable angina and non-ST-segment elevation myocardial infarction: summary article: a report of the American College of Cardiology/American Heart Association Task Force on Practice Guidelines (Committee on the Management of Patients With Unstable Angina). Circulation, v. 106, n. 14, p. 1893-900, 2002.

CHENIAUX, E. Manual de Psicologia. Rio de Janeiro-RJ: Gunabara Koogan, 2011.

CIRIC-ZDRAVKOVIC, S. V. et al. Anxiety in patients with acute coronary syndromes. The European Journal of Psychiatry, v. 28, n. 3, p.165-171, 2014.

CUNHA J. A. Manual da versão em português das Escalas Beck. São Paulo-SP: Casa do Psicólogo, 2001.

DESTÊRRO, V. Ansiedade. $2013 . \quad$ Disponível em: http://www.sbmfc.org.br/default.asp?site_Acao=MostraPagina\&PaginaId=508. 
Fonseca, F. A. H.et al.(2000). Modificações de hábitos de vida e outras opções terapêuticas. Revista da Sociedade de Cardiologia, 9(1), 1-7.

GUS, I., FISCHMANN, A., MEDINA, C. Prevalência de fatores de risco para doença arterial coronariana no estado do Rio Grande do Sul. Arquivo brasileiro de cardiologia, v. 78, n. 5, p. 478-483, 2001.

LEMOS, C., et al. Associação entre depressão, ansiedade e qualidade de vida após infarto do miocárdio. Psicologia: Teoria e Pesquisa, v. 24, n. 4, p. 471-476, 2008.

LIMA, L. R.; PEREIRA, S. V.; CHIANCA, T. C. Diagnósticos de enfermagem em pacientes póscateterismo cardíaco: contribuição de Orem. Rev Bras Enferm, v. 59, n. 3, p. 285-90, 2006.

MAGAlHÃES, C. C. et al. Tratado de cardiologia Socesp (3a ed.). São Paulo-SP: Manole, 2015.

MATOZINHOS F. P. et al. Saúde cardiovascular em população residente nas capitais brasileiras. Revista LatinoAm. Enfermagem, v.25. e. 2843, 1-9, 2017.

MACHADO, M. C.; PIRES, C. G. S.; LOBAO, W. M. Concepções dos hipertensos sobre os fatores de risco para a doença. Ciênc. saúde coletiva, Rio de Janeiro, v. 17, n. 5, p. 1357-1363, May 2012.

NASCIMENTO, L. F. C. Air pollution and cardiovascular hospital admissions in a medium-sized city in São Paulo State, Brazil. Brazilian Journal of Medical and Biological Research, v. 44, n. 7 , p. 720-724, 2011.

NASCIMENTO, L. F. C.; FRANCISCO, J. B. Particulate matter and hospital admission due to arterial hypertension in a medium-sized Brazilian city. Cadernos de Saúde Pública, v. 29, n. 8, p. 1565-1571, 2013.

NILSSON, U. et al. The effect of music intervention in relation togender during coronary angiographic procedures: a randomized clinical trial. Eur J Cardiovasc Nurs, v. 8, n. 3, p. 200-206, 2009.

PEDERSEN, S. S. et al. Course of anxiety symptoms over an 18-month period in exhausted patients post percutaneous coronary intervention. Psychosom Med., v. 70, n. 3, 349-355, 2008.

POLIWCZAK, A. R.; FUNT, D.; BRONCEL, M. The evaluation of discomfort and anxiety in the patient under going coronary angioplasty. Pol Merkur Lekarski, v. 35, n. 208), 202-204, 2013.

REZA, C. G., \& NOGUEIRA, M. S. O estilo de vida de pacientes hipertensos de um programa de exercício aeróbio: estudo na cidade de Toluca, México. Esc Anna Nery Rev Enferm,, v. 12, n. 2, 265-70, 2008.

ROEST, A. M. ET AL. Anxiety and risk of incident coronary heart disease: a metaanalysis. Journal of the American College of Cardiology, v. 56, n. 1, 38-46, 2010.

ROSSATO G, et al. Analysis of in-hospital complications related to cardiac catheterization. Rev Bras Cardiol Invas, v. 15, n. 1, p. 44-51, 2007. 
RUSSELL, M. et al. The conundrum of detectings table angina pectoris in the community setting. Nat Rev Cardiol., v. 7, n. 2, 106-13, 2010.

SANTOS, M. G., et al. Fatores de risco no desenvolvimento da aterosclerose na infância e adolescência. Arq Bras Cardiol, v. 90, n. 4, p.301-308, 2008.

SARDINHA, A. et al. Prevalência de transtornos psiquiátricos e ansiedade relacionada à saúde em coronariopatas participantes de um programa de exercício supervisionado. Arch. clin. Psychiatry. São Paulo, v. 38, n. 2, p. 61-65, 2011.

SCALON, P. et al. A report of the American College of Cardiology/American Heart Association Task Force on Practice Guidelines. J Am Coll Cardiol. v. 33, n. 6, p. 1756-1824, 2002.

SCHMIDT, D., et al. Ansiedade e depressão entre profissionais de enfermagem que atuam em blocos cirúrgicos. Revista da Escola de Enfermagem da USP. São Paulo, v.45, n. 2, p. 487-493, 2011.

VAN GESTEL, Y. R., et al. Type-D personality and depressive symptoms predict anxiety 12 months post-percutaneous coronary intervention. J Affect Disord, v. 103, n. 1-3, p. 197-203, 2007.

WORLD HEALTH ORGANIZATION. Ad herencetolong term therapies: evidence for action. Geneva, 2003. Disponível em: http://apps.who.int/iris/bitstream/10665/42682/1/9241545992.pdf

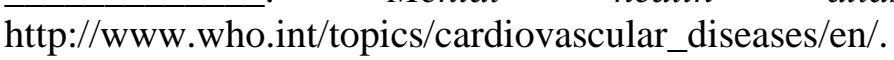

$$
\text { Mental health atlas, 2012. Disponível em: }
$$

Cardiovascular diseases (CVDs). 2016. Disponível em:

http://www.who.int/mediacentre/factsheets/fs317/en/

ZWIELESKI, G.; BUB, T. F. Identificação de Transtornos de Ansiedade em Pacientes com Suspeita de Doença Arterial Coronariana. Revista Brasileira de Cardiologia Rio de Janeiro, Rio de Janeiro, v. 27, n. 5, p. 311-313, 2014.

\section{Como citar este artigo (Formato ABNT):}

CANTONI, Eliandra de Paula; SILVEIRA, Michele Marinho da. Sintomas de Ansiedade, Angina Pectoris e Diagnóstico de Pacientes Submetidos ao Cateterismo Cardíaco. Id on Line Rev.Mult. Psic., 2018, vol.12, n.41, p.711-724. ISSN: 1981-1179.

Recebido: 04/07/2018

Aceito 13/07/2018 